\title{
Pioneer Venus Observations of Plasma and Field Structure in the Near Wake of Venus
}

\author{
J. G. Luhmann, ${ }^{1}$ C. T. Russell, ${ }^{1}$ L. H. Brace, ${ }^{3}$ H. A. Taylor, ${ }^{2}$ W. C. Knudsen, ${ }^{3}$ F. L. Scarf, ${ }^{1}$ \\ D. S. Colburn, ${ }^{5}$ AND A. Barnes 5
}

\begin{abstract}
Ionospheric plasma density depletions or 'holes' are observed by the Pioneer Venus orbiter in association with radial magnetic fields in the near wake of Venus. This report presents examples of the collected observations of these unexpected features of the Venus nightside ionosphere obtained by the Langmuir probe, magnetometer, ion mass spectrometer, retarding potential analyzer, plasma analyzer, and electric field experiments. The connection between plasma density depletions and temperature changes, changes in ion composition, plasma wave emissions, and magnetic fields with a substantial radial component is illustrated. Mechanisms that may be responsible for the formation and maintenance of holes are suggested.
\end{abstract}

\section{INTRODUCTION}

Localized depletions of plasma density or 'holes' in the nightside ionosphere of Venus were first mentioned by Brace et al. [1980] in an overview of the results of the Langmuir probe experiment on the Pioneer Venus Orbiter, although $\boldsymbol{H}$. A. Taylor et äl. [1979] had noted the structured nature of the altitude profiles of the nightside ion density earlier in the mission. These depletions in ionospheric plasma density were later found to be related to the presence of magnetic fields with substantial vertical components in a region slightly west of the midnight meridian [Brace et al., 1982]. In this letter the collected data from several representative cases are presented for the purpose of producing an overview of the available information on this as yet unexplained phenomenon.

\section{BACKGROUND}

Information about the Pioneer Venus orbiter and the experiments it carried can be found elsewhere (i.e., Science, 203(4382), 1979; Science, 205(4401), 1979; IEEE Trans. Geosci. Electron., GE-18, 1980). In considering the data that are presented here, one must keep in mind the bias imposed by the satellite orbit, which was near-polar, with $\sim 150-\mathrm{km}$ altitude periapsis and $\sim 75,000-\mathrm{km}$ apoapsis, and with periapsis fixed near $17^{\circ}$ north latitude. Also worth remembering is the fact that the magnetic field configuration around Venus depends on the conditions in the interplanetary plasma (i.e., see Spreiter and Stahara [1980]).

In addition to the Langmuir probe experiment [Krehbiel et al., 1980] and the magnetometer [Russell et al., 1980a], the ion mass spectrometer [Taylor et al., 1980a], the retarding potential analyzer [Knudsen et al., 1980], and the electric field detector [Scarf et al., 1980], detected distinctive signa-

\footnotetext{
${ }^{1}$ Institute of Geophysics and Planetary Physics, University of California Los Angeles, California 90024.

${ }^{2}$ NASA Goddard Space Flight Center, Greenbelt, Maryland 20771.

${ }^{3}$ Lockheed Missiles and Space Company, Palo Alto, California 94304.

${ }^{4}$ TRW Defense and Space Systems, Redondo Beach, California 90278.

${ }^{5}$ NASA Ames Research Center, Moffett Field, California 91103.

Copyright 1982 by the American Geophysical Union.
}

Paper number 2A1089.

0148-0227/82/002A-1089\$02.00 tures associated with the ionospheric holes. The plasma analyzer [Intriligator et al., 1980] provided measurements of the energetic plasma in the regions where the holes were observed by these other instruments. It should be mentioned that the neutral mass spectrometer data [Niemann et al., 1980] were also examined for unusual behavior in the presence of the nightside plasma density depletions, but none was found.

The plasma and field information obtained in the near wake of Venus by the aforementioned individual experiments can be briefly summarized as follows:

Langmuir probe observations. The Langmuir probe provides measurements of the thermal electron density and temperature. Localized decreases of up to several orders of magnitude in the thermal electron density relative to the normal nightside ionospheric density are observed in the probe data [Brace et al., 1980, 1982]. These 'holes' are found primarily from $2300 \mathrm{LT}$ to $0300 \mathrm{LT}$., in the latitude range $40^{\circ} \mathrm{N}$ to $20^{\circ} \mathrm{S}$, and at latitudes above $\sim 200 \mathrm{~km}$ in the antisolar region. The greatest depletions in density occur at the highest altitudes. Holes most often occur in pairs as seen in the time series of the ionospheric data for a given orbit. The temperature structure suggests the presence of two electron populations: an ionospheric component that is colder than usual and a hot possibly non-Maxwellian component that is most apparent in the center of the deepest holes and at high altitudes.

Magnetometer observations. The magnetometer generally detects small average magnetic fields ( $<10$ gammas) in the nightside ionosphere during conditions of undisturbed solar wind [Luhmann et al., 1981]. However, within a local time interval of a few hours in the region west of the midnight meridian, the magnetic field is frequently observed to increase to several 10 s of gammas. These magnetic fields exhibit an appreciable radial component that may reverse sign within the region of enhanced field. The region of enhanced field is sometimes separated into two sections by a region of low (normal) field strength, with the two sections of larger field having the same or opposite sign of radial component. Magnetic field structures of this type are present down to the altitude of periapsis $(\sim 150 \mathrm{~km})$ on occasion.

Ion mass spectrometer observations. The ion mass spectrometer measures thermal ion density and composition. Many different types of ion density depletions are observed between approximately 2100 and 0300 LT by the ion mass spectrometer, indicating the operation of ion loss processes 


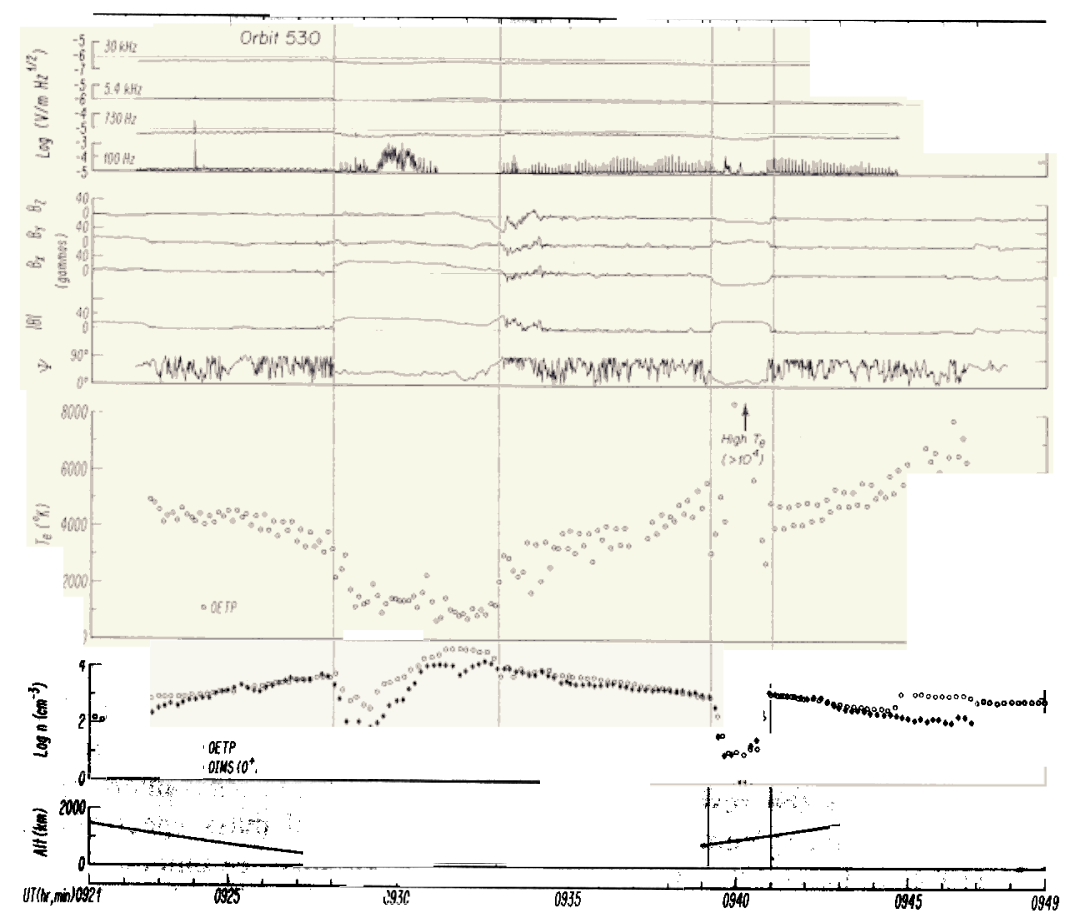

Fig. 1. Data obtained near periapsis on orbit 530 from the electric field, magnetometer, Langmuix probe (OETP), and Langmuir probe and ion mass spectrometer (OIMS) experiments in order from the top. The altitude above the surface is given at the bottom. The magnetic field components shown are in the solar ecliptic coordinate system; the angle $\psi$ is between the radial direction and the local magnetic vector (e.g., $\psi=0$ is radial). Note the location of the two 'square waves' of enhanced magnetic field (near periapsis) that are oriented at small angles to the radial, embedded in a typically weak, irregular nightside ionospheric field. The vertical lines delineate the approximate boundaries of the regions of enhanced magnetic field.

across a large sector of the nightside. Whereas ion distributions characteristic of the dayside ionosphere are often encountered, on other occasions the ionosphere is observed to be essentially 'depleted' down to the periapsis altitude [H. A. Taylor et al., 1979]. These completely 'depleted' cases, which are thought to be associated with loss processes operating throughout the wake region, will be discussed elsewhere. In less extreme 'transition' cases, such as those described in this report, the ionosphere exhibits pronounced structure, with localized density depletions ranging from a factor of 2 or 3 to orders of magnitude. This structure corresponds to the so-called 'holes' in the electron density measured by the Langmuir probe.

Within regions of density depletion, the ion composition when measurable (minimum densities are required for a reliable measurement) generally indicates the dominance of the light ions $\mathrm{H}^{+}$and $\mathrm{He}^{+}$in the presence of depletions in the usually dominant $\mathrm{O}^{+}, \mathrm{O}_{2}{ }^{+}$and molecular ions. The boundaries of the localized depletion regions are sometimes marked by a superthermal ion signature [see Taylor et al., $1980 \mathrm{~b}$ ]. This signature is interpreted as evidence of ion flow. The density within the holes is often too low to allow measurements of the thermal ion characteristics.

Retarding potential analyzer observations. The retarding potential analyzer (RPA) provides measurements of thermal ion density, temperature and bulk velocity. The RPA automatically selects a data sample only when the ion concentration is greater than approximately $10^{3} \mathrm{~cm}^{-3}$. Thus, $T_{i}$ data are not usually available in the holes except at the lowest altitudes where the density exceeds $10^{3} \mathrm{~cm}^{-3}$. Bulk flow data are also not obtainable in the holes. The total ion density data from the retarding potential analyzer agrees in general with the ion mass spectrometer results.

Electric field observations. The electric field detector provides information about the power at four frequencies: 30 $\mathrm{kHz}, 5.4 \mathrm{kHz}, 730 \mathrm{~Hz}$, and $100 \mathrm{~Hz}$. W. W. L. Taylor et al. [1979] first noted how, at low altitudes in the Venus shadow, impulsive events that fall into two classes occur: (1) broadband noise, which may be caused by gradient or current instabilities, and (2) whistler mode signals from atmospheric lightning. Scarf et al. [1980] showed that lightning is detected when the magnetic field is strong enough so that $f_{c} \gg 100$ $\mathrm{Hz}$ and when the field line at the point of the observations can be traced to a point of intersection with the planet. (Note that these conditions can be met within a hole but that they are met on other occasions also.)

Plasma analyzer observations. The plasma analyzer was designed for solar wind observations but has been found to be useful for detection of rapidly flowing ionospheric ions. Because of the many modes in the routine operation of this detector [Intriligator et al., 1980], and its low time resolution (the sampling cycles through energy and angle every $9 \mathrm{~min}$ compared with the time interval of $\sim 20-30 \mathrm{~min}$ that the orbiter takes to pass through the ionosphere), the detection of ion flows that occur on the spatial scale of the ionospheric holes is generally precluded. However, on occasion the appropriate measurement mode [cf. Mihalov and Barnes, 1982] is active while the spacecraft is within a hole, providing the possibility of detection of ion flow if present. Such 'snapshots' do not, of course, give information about the spatial scale of the region where flow is occurring or about the temporal behavior of the flow. 


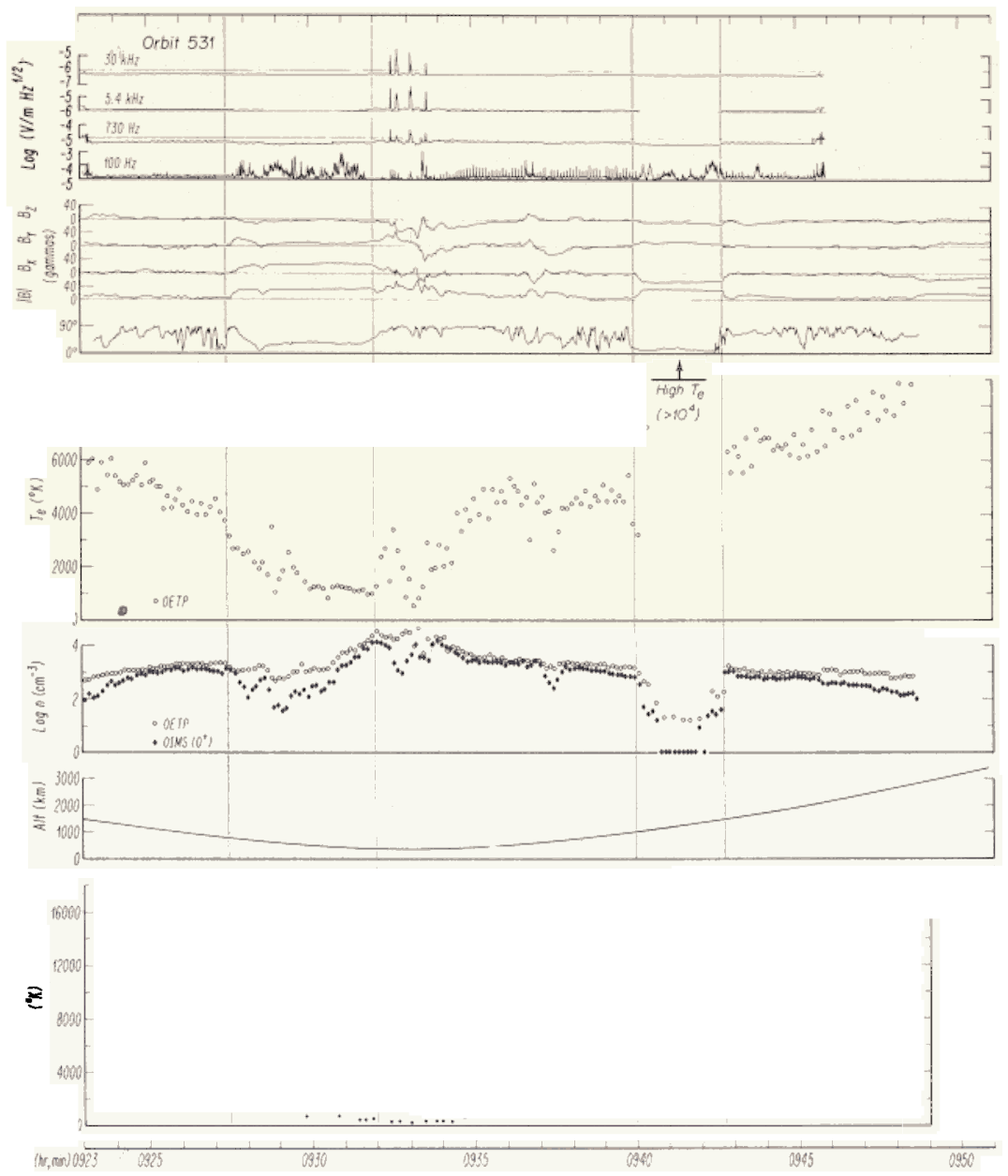

Fig. 2. Same as Figure 1 but for orbit 531, with the addition of ion temperature $\left(T_{i}\right)$ measurements from the retarding potential analyzer (bottom). Note the similarity of this orbit to orbit 530 (Figure 1), which occurred 24 hours earlier.

\section{OBSERVATIONS}

Collected data from four of the aforementioned six experiments (the electric field detector, the magnetometer, the Langmuir probe (OETP) and the ion mass spectrometer (OIMS)) for three characteristic examples of Pioneer Venus near-wake passes are given in Figures 1, 2 and 4; Figures 2 and 4 each include observations from one additional experiment. In these three figures, the top panel shows the electric field data from the four channels of the electric field detector; the second set of panels shows the magnetic field components in the solar ecliptic coordinate system, total field magnitude $|B|$ and $\psi$, the angle between the radial direction and the observed magnetic vector; the third panel from the top gives the electron temperature $T_{e}$ obtained by the Langmuir probe; the fourth panel contains the electron density from the Langmuir probe measurement and the $\mathrm{O}^{+}$ density $\left(\mathrm{O}^{+}\right.$is the predominant ion above $200-\mathrm{km}$ altitude) from the ion mass spectrometer data, and the altitude of the spacecraft is shown in the fifth panel. Figure 2 includes an additional panel showing measurements of $T_{i}$ that were available from the retarding potential analyzer for orbit 531 .

In Figure 1, which shows data from orbit 530, there are two clearly defined depletions or holes in the plasma density centered at $\sim 0930$ UT and $\sim 0940$ UT. Both holes occur where the magnetic field magnitude increases. The small values of $\psi$ indicate the field's nearly radial direction. The first electron density depletion disappears at the lowest altitudes even though the magnetic field structure persists. The electron temperature decreases at the boundaries of the holes but is higher than usual in the central region of the lowaltitude hole and throughout most of the high-altitude hole. The inequality of the electron and $\mathrm{O}^{+}$densities within the holes indicates that ions other than $\mathrm{O}^{+}$are present in significant concentrations. The plasma analyzer was not in the appropriate mode for detecting ion flow during this period. Impulsive $100-\mathrm{Hz}$ plasma waves are detected within the holes. These electric field data also show a fairly rare and unexplained $100-\mathrm{Hz}$ signal with extremely strange characteristics suggesting a spacecraft origin. The very regular set of $100 \mathrm{~Hz}$ impulses covering the intervals 0933 to 0939 and 0941 to 0945 were detected only when the $E$ field antenna was directly aligned with the anti-solar direction, producing modulation at twice the spin frequency. There is presently no explanation for these observations since the spacecraft was in darkness and vectors associated with the spacecraft velocity and the $B$ field direction could not directly produce phenomena oriented along an anti-solar axis.

Figure 2 shows the corresponding data from orbit 531. Again two well-defined holes are observed centered about 


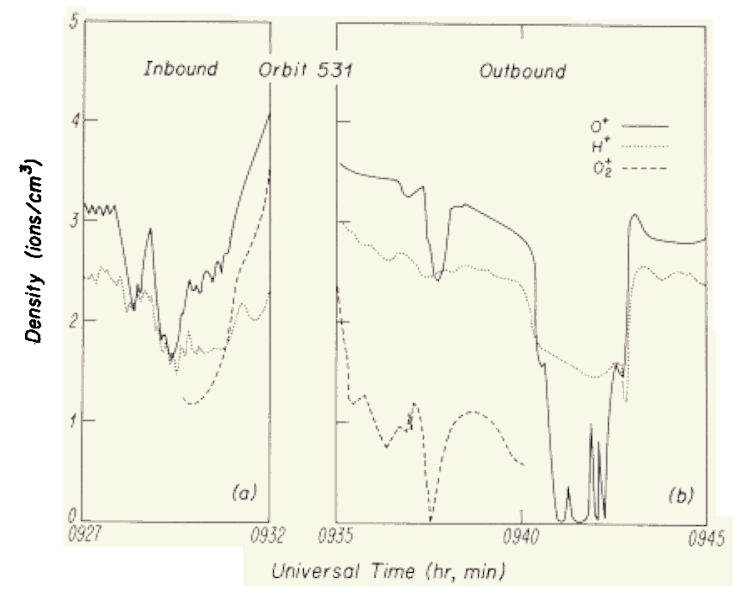

Fig. 3. Ion composition during sections of orbit 531 near the holes as measured by the ion mass spectrometer.

0931 UT and 0941 UT, in conjunction with apparent ion composition changes, impulsive plasma waves at $100 \mathrm{~Hz}$ and radial magnetic fields. As in the case of orbit 530 , the entry into the first hole coincides with the onset of the radial magnetic field, but the exit from the hole at low altitudes is not clearly defined, although the radial character of the field ceases at about 0932. In contrast, the hole encountered around 0940 UT is clearly defined by the magnetic field structure. The bottom panel shows the retarding potential analyzer ion temperature data for this pass. The large range of $T_{i}$ is notable; $T_{i}$ is large when the spacecraft is outside of the holes and above approximately $200-\mathrm{km}$ altitude, while inside the inbound hole of orbit $531, T_{i}$ is low. In general, the behavior of $T_{i}$ is consistent with that measured elsewhere at comparable altitudes [Miller et al., 1980; Knudsen et al., 1979]. The ion temperature may change in the holes but there are no data in the lowest density regions to confirm this. Again, the plasma analyzer was not in the appropriate mode for the detection of ion flow during this period. Details of the ion composition as measured by the ion mass spectrometer during selected sections of orbit 531 near the holes are given in Figure 3. Here it is demonstrated that the relative abundances of the ionospheric constituents are different in the regions where plasma density depletions are located than in the surrounding normal ionosphere. As mentioned above, at the high altitudes, $\mathrm{H}^{+}$can replace $\mathrm{O}^{+}$as the dominant ion. However, the composition appears unaffected near periapsis even when the radial magnetic field structure extends through periapsis (see Figure $3 a$ ).

Figure 4 illustrates the data obtained during the periapsis pass of orbit 526. This orbit is particularly interesting because the plasma density, shown by the open circles, is only slightly depleted at the high-altitude edges of a region of radial magnetic field $\left(\psi \simeq 0^{\circ}\right)$ surrounding periapsis at $\sim 0927$ UT. (The decrease in the $\mathrm{O}^{+}$density occurs when the spacecraft enters the altitude range where $\mathrm{O}_{2}{ }^{+}$is the dominant ion.) However, other features that are usually associated with holes are observed from $\sim 0925$ to 0931 UT: nearly radial magnetic field, impulsive plasma wave emissions at $100 \mathrm{~Hz}$, and a change in electron temperature. During orbit 526 , the plasma analyzer happened to cycle through the appropriate mode while the spacecraft was in the region of radial magnetic field and ion flow was detected (the time of flow detection is indicated by the arrow in Figure 4). If the ion is $\mathrm{O}_{2}{ }^{+}$, the flow speed relative to the planet is $7.5 \mathrm{~km} / \mathrm{s}$. The detector angle during the measurement indicates that the flow had a downward component of $3.6 \mathrm{~km} / \mathrm{s}$.

\section{Discussion}

Although certain aspects of the phenomenon represented by Figures 1, 2, and 4 were included in the previous discussions of Brace et al. [1982] and Luhmann et al. [1981], the collected data shown here provide a more complete picture for the theorists' interpretation.

As illustrated by Figure 4, a quasi-vertical magnetic field structure, such as that occurring between $\sim 0925$ and 0931 UT on orbit 526, may be present near periapsis without an apparent depletion in the electron density. This is in contrast to the outbound (right-hand side) holes in Figures 1 and 2 which show a plasma density decrease throughout the magnetic structure. The inbound (left-hand side) holes in Figures 1 and 2 perhaps explain this in terms of an altitude effect. In the high-altitude sections of the inbound magnetic field enhancements in orbits 530 and 531 a plasma density hole is apparent, but the density hole disappears on the approach to periapsis in spite of the fact that the magnetic field persists. On the basis of these observations, it can be inferred that whatever mechanism causes the localized plasma density depletion is ineffective below an altitude of $\sim 200 \mathrm{~km}$ [Brace et al., 1982].

It is also apparent from Figures 1 and 2 that the outbound holes, which are observed at a higher altitude than the inbound holes, are much more depleted and hotter than the inbound holes. Moreover, the magnetic field direction in the outbound holes is closer to the radial direction than the field in the inbound holes.

The event in the electric field data at 0932:30 to 0934:00 during orbit 531 (Figure 2) is believed to be current-driven
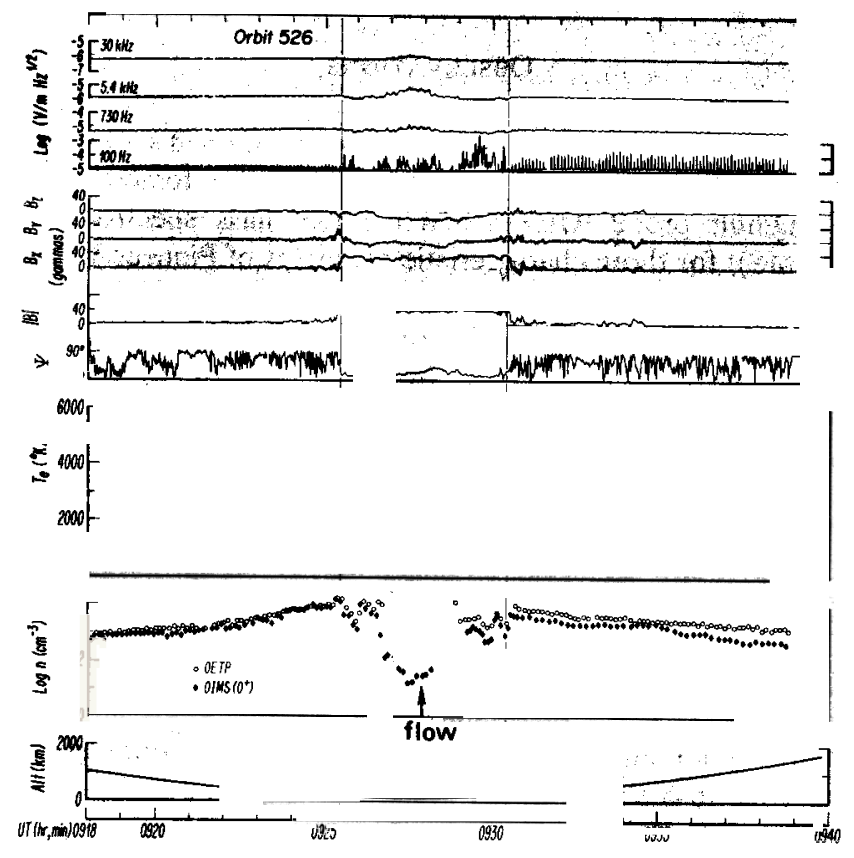

Fig. 4. Same as Figures 1 and 2 for orbit 526. The apparent depletion in the $\mathrm{O}^{+}$concentration near periapsis occurs where molecular $\mathrm{O}_{2}^{+}$(not shown) becomes dominant. The arrow shows where the plasma analyzer detected ion flow (see text). 


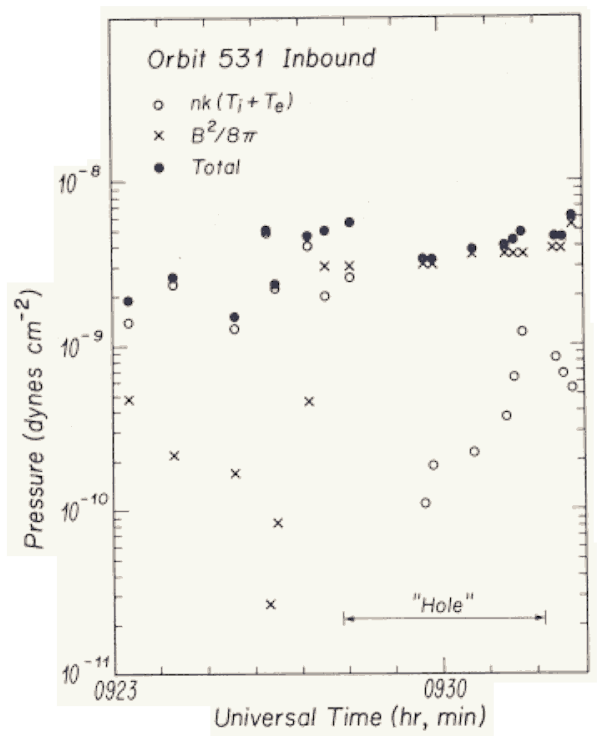

Fig. 5. Comparison of thermal pressure $n k\left(T_{e}+T_{i}\right)$ and magnetic pressure $|B|^{2} / 8 \pi$ within the inbound hole of orbit 531 .

electrostatic turbulence similar to that detected in the earth's polar cusp and auroral region [Fredricks et al., 1973; Scarf et al., 1973]. The impulsive $100-\mathrm{Hz}$ noise detected within the holes (which was also seen on orbits 530 and 526) is interpreted as lightning, although the possibility that this noise is associated with the hole formation process cannot be excluded.

The measurements of $T_{i}$ for the inbound hole of orbit 531 (Figure 2) allow the comparison throughout the structure of magnetic pressure $|B|^{2} / 8 \pi$ and thermal pressure $n k\left(T_{e}+T_{i}\right)$, where $n$ is plasma (electron) density and $k$ is Boltzmann's constant. The result, shown in Figure 5, indicates that pressure balance can occur through the regions of holes, as inferred by Brace et al. [1982] by using electron and magnetic field data only. Moreover, the value of $\beta$, where $\beta$ is the ratio of thermal to magnetic pressure, changes from $\beta>1$ outside the holes to $\beta<1$ inside the holes.

An additional point that may be relevant to the understanding of the holes, considering the observation of the ion flow by the plasma analyzer during orbit 526, is the observation of heavy ions streaming away from Venus in the distant wake at the Pioneer Venus apoapsis distance of $\sim 10$ planetary radii [Mihalov and Barnes, 1982]. Ionospheric $\mathrm{O}^{+}$ions in the magnetotail may originate in the nightside holes region, although it appears that at least some ionospheric ions are picked up by the solar wind near the terminator.

One interpretation of the plasma holes and the related magnetic field structures has been suggested by Brace et al. [1982]. These authors formulate a picture in which field lines initially draped over the dayside ionosphere and connected at both ends to the magnetosheath flow are dragged over the terminator by ionospheric convection and magnetic tension into the nightside ionosphere on their way to the magnetotail. The vertical magnetic field prevents, at altitudes above $\sim 200 \mathrm{~km}$, the horizontal flow from the dayside toward the antisolar point that usually supplies the nightside ionosphere. The flow at low altitudes is not as strongly affected by the magnetic field because ion-neutral collisions permit perpendicular transport of the ions there. Within the region of vertical field and low ionospheric plasma density, hot magnetotail electrons have access to low altitudes, thus providing the observed hot component. The ionosphere within the holes is cool relative to that outside because heat conduction into the cool low altitude ionosphere is permitted by the vertical field geometry, while horizontal heat transport is inhibited. The details of this model are left as an open question.

Another interpretation of the holes has been offered by Grebowsky and Curtis [1981], who compare the Venus nightside ionosphere with the auroral ionosphere. The formation of the plasma holes is attributed to the existence of a parallel electric field in the region of the vertical magnetic field. In this context it should be mentioned that $\mathrm{T}$. Gombosi (personal communication, 1981) finds evidence that energetic electron precipitation helps maintain the nightside ionosphere as suggested by Gringauz et al. [1977].

A related interpretation has been formulated by $R$. E. Hartle (personal communication, 1982) based on the ion composition measurements within holes. Polar wind-like outflow of $\mathrm{H}^{+}$(and perhaps of $\mathrm{O}^{+}$) is suggested as the reason for the altitude distribution of $\mathrm{H}^{+}$and $\mathrm{O}^{+}$below $200 \mathrm{~km}$ where the plasma is still collisional. The outflow is related to the evacuation of plasma, at the higher altitudes, by parallel electric fields. Enhanced diffusion along the radial magnetic field [Scarf et al., 1980] may also play a role in the escape of ionospheric plasma in the holes.

Elements of all of the aforementioned explanations are probably appropriate. As Brace et al. [1982] noted, horizontal flow from the dayside will be impeded by the vertical field structure above $\sim \mathbf{2 0 0} \mathrm{km}$. Although the depletion of plasma by outflow might therefore seem unnecessary since the supply is cut off, with ionospheric plasma flowing horizontally around the obstacles presented by the vertical field structures, the composition data indicate outflow is occurring [Hartle et al., 1982]. Electron precipitation within the vertical field structures, related to parallel electric fields driven by magnetotail processes, will cause heating of the ionosphere as observed in the terrestrial aurorae as pointed out by Grebowsky and Curtis [1981]. The plasma emissions seen in the electric field data may result from this activity. Adjustment of the scale heights of the ionospheric constituents within the vertical field structures to the heating and the boundary conditions imposed from above must follow.

One problem that remains outstanding is the origin and evolution of the near wake magnetic field structures. A connection between the magnetosheath field, formed by interplanetary field draping around the ionosphere, and nightside ionospheric field directions in the regions of radial field was suggested by the magnetic field data [Luhmann et al., 1981]. However, the possibility that the flux tubes of the radial field structures are the magnetotail lobes arising from a weak intrinsic field [e.g., see Dolginov et al., 1978] cannot be overlooked except that this interpretation is not supported by the statistical analysis of Russell et al. [1980 b] that showed no observational evidence of measurable planetary magnetism. In any case, the data do not give very complete information about the low-altitude magnetic structure or about the three-dimensional shape of the magnetic structures (i.e., are they isolated columnar tubes of flux or large regions with irregularly shaped boundaries) because of the combination of sampling bias by the orbit and the variability in the magnetosheath field direction from orbit to orbit. 
Recently, examination of nightside data obtained during the extended mission, in which periapsis is being allowed to rise, has revealed some information about how these structures evolve with altitude. The magnetic geometry is similar to the structure in the high-altitude (outbound) holes seen in orbits 530 and 531 (Figures 1 and 2), with occasional intrusions of irregularly oriented magnetosheath fields in between the holes. The ability of a hole to maintain its vertical geometry through the topside ionosphere is apparent. Further extended mission data, obtained in the nightside as the Pioneer Venus orbiter rises above the ionosphere, are expected to reveal the fate of these magnetic structures as they merge with the magnetotail fields from the magnetosheath. The particle characteristics at these higher altitudes may help test the various ideas mentioned above concerning the interpretation of the structure of the Venus near-wake.

Acknowledgments. The authors acknowledge discussions with other PVO investigators, including H. B. Niemann, J. D. Mihalov, P. A. Cloutier, R. C. Elphic, R. E. Hartle, A. Nagy, and T. Cravens and the help of G. McLean, N. Cline, H. Herbert, and R. Warniers in the organization and presentation of the collected data. Support was provided by NASA contract NAS 2-9491.

The Editor thanks the two referees for their assistance in evaluating this paper.

\section{REFERENCES}

Brace, L. H., R. F. Theis, W. R. Hoegy, J. H. Wolfe, J. D. Mihalov, C. T. Russell, R. C. Elphic, and A. F. Nagy, The dynamic behavior of the Venus ionosphere in response to solar wind interactions, J. Geophys. Res., 85, 7663, 1980.

Brace, L. H., R. F. Theis, H. G. Mayr, S. A. Curtis, and J. G. Luhmann, Holes in the nightside ionosphere of Venus, $J$. Geophys. Res., 87, 199, 1982.

Dolginov, Sh. Sh., L. N. Zhuzgov, V. A. Sharova, and V. B. Buzin, Magnetic field and magnetosphere of the planet Venus, Kosm. Issled., 16, 870, 1978.

Fredricks, R. W., F. L. Scarf, and C. T. Russell, Field-aligned currents, plasma waves and anomalous resistivity in the disturbed polar cap, J. Geophys. Res., 78, 2133, 1973.

Grebowsky, J. M., and S. A. Curtis, Venus nightside ionospheric holes: The signatures of parallel electric field acceleration regions?, Geophys. Res. Lett., 8, 1273, 1981.

Gringauz, K. I., M. I. Verigin, T. K. Breus, and T. Gombosi, Electron fluxes measured on board Venera 9 and 10 in the optical umbra of Venus: Main ionization source in Venus' nighttime ionosphere, Dokl. Akad. Nauk SSSR, 232, 1039, 1977.

Intriligator, D. S., J. H. Wolfe, and J. D. Mihalov, The Pioneer Venus orbiter plasma analyzer experiment, IEEE Trans. Geosci. Remote Sensing, GE-18, 39, 1980.

Knudsen, W. C., K. Spenner, R. C. Whitten, J. R. Spreiter, K. L. Miller, and V. Novak, Thermal structure and energy influx to the day and nightside Venus ionosphere, Science, 205, 105, 1979.

Knudsen, W. C., K. Spenner, J. Bakke, and V. Novak, Pioneer Venus orbiter planar retarding potential analyzer plasma experi- ment, IEEE Trans. Geosci. Remote Sensing, GE-18, 54, 1980.

Krehbiel, J. P., L. H. Brace, R. F. Theis, J. R. Cutler, W. H. Pinkus, and R. B. Kaplan, Pioneer Venus orbiter electron temperature probe, IEEE Trans. Geosci. Remote Sensing, GE-18, 49, 1980.

Luhmann, J. G., R. C. Elphic, C. T. Russell, J. A. Slavin, and J. D. Mihalov, Observations of large scale steady magnetic fields in the nightside Venus ionosphere and near wake, Geophys. Res. Lett., $8,517,1981$.

Mihalov, J. D., and A. Barnes, The distant interplanetary wake of Venus: Plasma observations from Pioneer Venus, J. Geophys. Res., in press, 1982.

Miller, K. L., W. C. Knudsen, K. Spenner, R. C. Whitten, and V. Novak, Solar zenith angle dependence of ionospheric ion and electron temperatures and density on Venus, J. Geophys. Res., $85,7759,1980$.

Niemann, H. B., J. R. Booth, J. E. Cooley, R. E. Hartle, W. T. Kasprzak, N. W. Spencer, S. H. Way, D. M. Hunten, and G. R. Carignan, Pioneer Venus, orbiter neutral gas mass spectrometer experiment, IEEE Trans. Geosci. Remote Sensing, GE-18, 60, 1980.

Russell, C. T., R. C. Snare, J. D. Means, and R. C. Elphic, Pioneer Venus orbiter fluxgate magnetometer, IEEE Trans. Geosci. Remote Sensing, GE-18, 32, $1980 a$.

Russell, C. T., R. C. Elphic, and J. A. Slavin, Limits on the possible intrinsic magnetic field of Venus, J. Geophys. Res., 85, 8319, $1980 b$.

Scarf, F. L., R. W. Fredricks, C. T. Russell, M. Kivelson, M. Neugebauer, and C. R. Chappell, Observation of a current-driven plasma instability at the outer zone-plasma sheet boundary, $J$. Geophys. Res., 78, 2150, 1973.

Scarf, F. L., W. W. L. Taylor, and P. F. Virobik, The Pioneer Venus orbiter plasma wave investigation, IEEE Trans. Geosci. Remote Sensing, GE-18, 36, 1980.

Scarf, F. L., W. W. L. Taylor, C. T. Russell, and L. H. Brace, Lightning on Venus: Orbiter detection of whistler signals, $J$. Geophys. Res., 85, 8158, 1980.

Spreiter, J. R., and S. S. Stahara, Solar wind flow past Venus: Theory and comparisons, J. Geophys. Res., 85, 7715, 1980.

Taylor, H. A., Jr., H. C. Brinton, S. J. Bauer, R. E. Hartle, P. A. Cloutier, R. E. Daniell, Jr., and T. M. Donahue, Ionosphere of Venus: First observations of day-night variations of the ion composition, Science, 205, 96, 1979.

Taylor, H. A., Jr., H. C. Brinton, T. C. G. Wagner, B. H. Blackwell, and G. R. Cordier, Bennett ion mass spectrometers on the Pioneer Venus bus and orbiter, IEEE Trans. Geosci. Remote Sensing, GE-18, 44, 1980a.

Taylor, H. A., Jr, H. C. Brinton, S. J. Bauer, R. E. Hartle, P. A. Cloutier, and R. E. Daniell, Jr., Global observations of the composition and dynamics of the ionosphere of Venus: Implications for the solar wind interaction, J. Geophys. Res., 85, 7765, $1980 b$.

Taylor, W. W. L., F. L. Scarf, C. T. Russell, and L. H. Brace, Absorption of whistler mode waves in the ionosphere of Venus, Science, 205, 112, 1979.

(Received January 5, 1982; revised July 6,1982 ; accepted July 20,1982 .) 